\title{
Multimodality treatment in hormone-refractory prostate cancer patients with bone metastases
}

\author{
Marnix G. E. H. Lam • Bernard A. Zonnenberg
}

Published online: 26 April 2008

(C) The Author(s) 2008

\section{Dear Sir,}

We agree with the interesting points made by Koutsikos et al. They confirm the feasibility of the combined use of bone-seeking radiopharmaceuticals and bisphosphonates and highlight the potential benefit of this combined treatment. We would like to stress the importance to study multimodality treatment strategies in well-designed clinical trials. These trials should evaluate the multidimensional character of pain, as well as survival, and they should incorporate imaging modalities for response stratification.

Bone-seeking radiopharmaceuticals are indicated for the relief of bone pain in patients with multiple painful osteoblastic metastases. Several new approaches are being studied not only to improve treatment efficacy related to pain response but also to extend efficacy by improving overall survival. As a single treatment modality, treatment at short intervals has shown to be beneficial with regard to pain palliation and survival [1]. However, most progress has been made with combinations of treatment modalities, for example, bone-seeking radiopharmaceuticals and chemotherapy [2].

This reply refers to the letter to the editor at http://dx.doi.org/10.1007/ s00259-008-0759-4.

M. G. E. H. Lam $(\bowtie) \cdot$ B. A. Zonnenberg

Department of Nuclear Medicine,

University Medical Center Utrecht,

P.O. Box 85500, 3508 GA Utrecht, The Netherlands

e-mail: M.Lam@umcutrecht.nl

B. A. Zonnenberg

Department of Internal Medicine, UMC Utrecht,

Utrecht, The Netherlands
In a recent publication, we showed the feasibility of ${ }^{153} \mathrm{Sm}$-EDTMP combined with zoledronic acid in hormonerefractory prostate cancer patients [3]. The combination of these two phosphate-based pharmaceuticals does not lead to competition in uptake at the level of the calcified bone matrix, nor does it lead to unacceptable toxicity. As a phase I study, it was not designed to study efficacy. However, as an illustration of potentially increased efficacy, two patients showed a complete remission of pain and a remarkable decline in prostate-specific antigen levels over pretreatment values $(-71 \%$ and $-43 \%)$, and markers of bone metabolism $(-65 \%$ and $-90 \%)$ over a period of almost 6 months. These results suggest a clinical benefit. But, further research is needed to explore the possible benefit of bone-seeking radiopharmaceuticals in combination with bisphosphonates, preferably in phase II and phase III randomized controlled settings.

In the past, our center performed a double-blind, placebo-controlled, randomized trial to study the efficacy of ${ }^{186}$ Re-HEDP compared to placebo [4]. Pain relief was assessed using an electronic diary containing questions reflecting the multidimensional character of pain. Daily pain assessment was mandatory because it fluctuated day by day. Besides a visual analog scale for pain and the assessment of daily activities, a medication index score was also registered. This complex method of evaluation showed a significant decrease of pain in the treated population. Besides this, the study illustrated the difficulty of pain response as an endpoint and the necessity of a welldesigned method for pain evaluation. Although a survival benefit could not be detected in this study using ${ }^{186} \mathrm{Re}$ HEDP as a single modality treatment, survival should be studied in future trials focusing on the efficacy of bone-seeking radiopharmaceuticals in combination with bisphosphonates. 
Fig. 1 Proposed treatment regimen in hormone-refractory prostate cancer patients
CONCOMMITANT MEDICATION (i.e. hormonal treatment, biphosphonates )

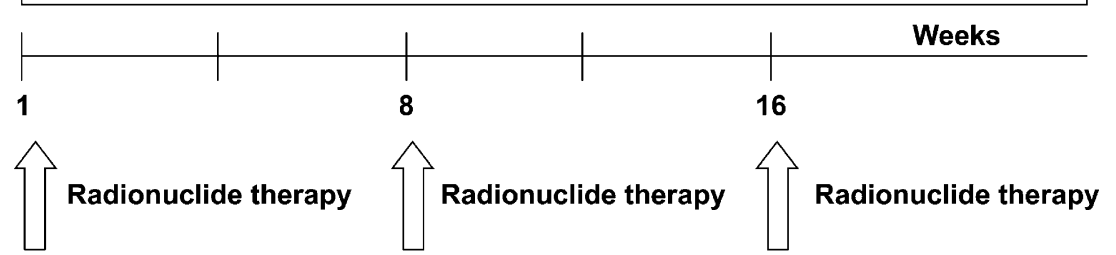

RADIATION MODIFIER (i.e. chemotherapy)
Several clinicians have postulated the multimodality approach for the treatment of hormone-refractory prostate cancer patients with painful osseous metastases. Combinations of multiple single treatment modalities may lead to an improvement of clinical benefit beyond an additive effect alone. From all available single-agent modalities, docetaxel is currently the first choice because of its proven efficacy on patients' survival [5]. Its toxicity is considerable, but responders show a clear benefit with regard to quality of life and survival. A combination of radiopharmaceuticals and chemotherapy might be an interesting therapeutical option. Besides the clinical benefit of each pharmaceutical separately, the radiation-sensitizing effect of chemotherapy may lead to synergy and a further improvement of clinical benefit. The combined use of chemotherapy as a radiosensitizer is well known in the field of external beam radiotherapy but must still be explored in the field of radionuclide therapy. Potential radiation modifiers may be docetaxel or other chemotherapeutics (such as platinum compounds or 5-FU). In hormone-refractory prostate cancer patients, continuous treatment with hormones and bisphosphonates in combination with periodical administrations of a bone-seeking radiopharmaceutical, modified by chemotherapy, may prove to be an effective regimen (Fig. 1). Such a regimen benefits from (1) the effects of repeated treatment with radiopharmaceuticals, bisphosphonates, and chemotherapy as single-agent modalities and (2) the radiation-sensitizing effects of chemotherapy. Different possible strategies should be studied in the future.

Last but not least, one of the greatest challenges in the field of nuclear oncology and oncology in general will be the selection of responders and nonresponders. Especially in hormone-refractory prostate cancer, a minority of treated patients show clinical benefit. It will prove to be beneficial to patients, as well as cost-effective, to recognize those who respond to the proposed treatment regimen. Molecular imaging may play a crucial role in identifying these subjects. In hormone-refractory prostate cancer patients, several tracers are being developed and studied for positron emission tomography imaging [6]. Stratification of patients and the development of new treatment strategies will lead to improvement of efficacy with improved overall survival.

Open Access This article is distributed under the terms of the Creative Commons Attribution Noncommercial License which permits any noncommercial use, distribution, and reproduction in any medium, provided the original author(s) and source are credited.

\section{References}

1. Palmedo H, Manka-Waluch A, Albers P, Schmidt-Wolf IG, Reinhardt M, Ezziddin S, et al. Repeated bone-targeted therapy for hormone-refractory prostate carcinoma: randomized phase II trial with the new, high-energy radiopharmaceutical rhenium-188 hydroxyethylidenediphosphonate. J Clin Oncol. 2003;21:2869-75.

2. Tu SM, Millikan RE, Mengistu B, Delpassand ES, Amato RJ, Pagliaro LC, et al. Bone-targeted therapy for advanced androgenindependent carcinoma of the prostate: a randomised phase II trial. Lancet. 2001;357:336-41.

3. Lam MG, Dahmane A, Stevens WH, van Rijk PP, de Klerk JM, Zonnenberg BA. Combined use of zoledronic acid and $153 \mathrm{Sm}-$ EDTMP in hormone-refractory prostate cancer patients with bone metastases. Eur J Nucl Med Mol Imaging. 2008;35:756-5.

4. Han SH, de Klerk JM, Tan S, het Schip AD, Derksen BH, van Dijk A, et al. The PLACORHEN study: a double-blind, placebocontrolled, randomized radionuclide study with (186)Re-etidronate in hormone-resistant prostate cancer patients with painful bone metastases. Placebo Controlled Rhenium Study. J Nucl Med. 2002;43:1150-6.

5. Tannock IF, de Wit R, Berry WR, Horti J, Pluzanska A, Chi KN, et al. Docetaxel plus prednisone or mitoxantrone plus prednisone for advanced prostate cancer. N Engl J Med. 2004;351:1502-12.

6. Pantaleo MA, Nannini M, Maleddu A, Fanti S, Ambrosini V, Nanni $\mathrm{C}$, et al. Conventional and novel PET tracers for imaging in oncology in the era of molecular therapy. Cancer Treat Rev. 2008; $34: 103-21$ 